II.

Aus dem Pharmakologischen Institut der Universität Freiburg i. Br.

Eine neue Methode zur Bestimmung des Broms in kleinsten Mengen.

Von

Ernst Oppenheimer.

Bei meinen Untersuchungen (siehe die folgende Abbandlung), die sich mit der spezifischen Wirkang der Bromidionenwirknng beschäftigten, war ich auf eine quantitative Methode des Bromnachweises angewiesen, die besonders milligramatische Mengen mit möglichst großer Genauigkeit zu erfassen gestattete. Unter den bisher bekannten erwies sich keine für die speziellen Zwecke geeignet.

Die älteste Brombestimmung, die die Halogene als Silberhalogenide fallt und mit dem Chlorstrom $\mathrm{AgBr}$ in $\mathrm{AgCl}$ umwandelt, and bei der aus dem Differenzgewicht die Menge des Broms zu berechnen ist, ist zwar zweifellos sehr exakt, aber zu umständlich und zeitraubend, nnd für kleinere Mengen zu ungenau. Sie ist von den wenigsten biologisch arbeitenden Autoren benutzt worden. Für die Methode von Jannasch1), der das Brom mit Kaliumpermanganat and Essigsäure von den übrigen Halogenen trennt, gilt das gleiche. Am häufigsten angewandt scheint die Methode von Berglund 2), wie sie Bönniger ${ }^{3}$ ) und Frey ${ }^{4}$ ) modifiziert haben. Die Methodik der Analyse von v. $W^{y^{5}}{ }^{5}$ hat Bernoulli $\left.{ }^{6}\right)$ bereits einer Kritik unterzogen und gefunden, daß sie bei gleichzeitiger Anwesen-

1) Jannasch, zitiert nach Classen, Handb. d. quant. chem. Analyse. Stuttgart 1912, Enke.

2) Berglund, Methode, um Chlor und Brom qualitativ zu scheiden. Zeitschr. f. analyt. Chem. 1885, Bd. 24, S. 184.

3) Bönniger, Die Substituierung des Chlors durch Brom im tierischen Körper. Zeitschr. f. exp. Path. a. Ther. 1907, Bd. 4, S. 414.

4) Frey, Ernst, Die Ursache der Bromretention; Ein Vergleich der Bromund Chlorausscheidung durch die Niere. Ebenda 1910, Bd. 8, S. 29.

5) v. Wy B, Über das Verhalten der Bromsalze im menseblichen und tierischen Organismus. Arch. f. exp. Path. u. Pharm. 1906, Bd. 55, S. 263.

6) Bernoulli, Untersuchungen über die Wirkung der Bromşalze. Ebenda 1913, Bd. 73, S. 355. 
heit von Cl wesentlich ungenauere Werte gibt als die Berglundsche. Ganz frei von diesem Fehler - da die Abspaltbarkeit ja nur graduell sich unterscheidet - ist aber auch die Berglundsche Analyse nicht. Ich bin selbst verschiedentlich anf das Bergl undsche Verfahren zurïckgekommen und konnte feststellen, daß ihre Verwertbarkeit stark von dem jeweiligen Verhältnis $\mathrm{Cl}: \mathrm{Br}$ abhängt, daß ferner die Stärke und Dauer des Luftstroms, mit dem das durch Kaliumpermanganat und -pyrosulfat freigesetzte $\mathrm{Br}$ durch die JK-Vorlagen geführt wird, für das Ergebnis von wesentlicher Bedeutung ist. Mit dem von Bernoulli angegebenen Optimum von 30 Minuten bin ich nicht ansgekommen. Vorversuche bestimmten mich, die Reaktion erst nach $3 / 4-1$ Stunde als beendet anzusehen. Daß ich anstatt mit. $50 \mathrm{ccm}$ einer 10- bzw. 5- und $2 \%$ igen JK-Lösung bei den derzeitigen Jodpreisen, auch mit ëiner Vorlage mit viel schwücherer Jodkalilösung, die außerdem noch eine tiberschlagsweise berechnete $\mathrm{Na}_{2} \mathrm{~S}_{2} \mathrm{O}_{3}$ Lösung enthielt, befriedigende Resultate erzielte, sei nebenbei bemerkt. Ein weiterer Umstand setzt der Berglundschen Analysenmethode eine Grenze. Für Brommengen unter $20 \mathrm{mg}$ werden die Fehler nach meinen Erfahrungen, je bromärmer das Analysenmaterial wird, immer größer.

DaB die genannten Bestimmungsarten den Anforderungen für biologische Fragestellungen nicht vollauf gentigen, hat schon Win sche ${ }^{1}$ ) empfunden und aus dem gleichen Bedurfnis heraus eine nene $\mathrm{Me}$ thode ${ }^{2}$ ) ersonnen und angegeben. Er ging von der Entdeckung Guareschis ${ }^{3}$ ) aus, der 1913 feststellte, daß durch $\mathrm{SO}_{2}$ entfärbtes Fuchsin (Schiffsches Reagens) durch $\mathrm{Br}$ tiefblauviolett gefärbt wird. Der qualitative Ausfall dieser Reaktion - und das schien das praktisch bedeutungsvollste an ihr - wird nach Aussage des Entdeckers durch die Anwesenheit von $\mathrm{Cl}$ und $\mathrm{J}$ so gat wie gar nicht gestört. Bei seinen rein qualitativen Untersuchungen ging Guareschi so vor, daß er das durch Oxydationsmittel entwickelte $\mathrm{Br}$ auf einen im

1) Wünsche, Eine neue Methode zur Bestimmang von Brom. Arch. f. exp. Path. u. Pharm. 1919, Bd. 84, S. 328.

2) Mit der Methode Autenrieths4) hat Wünsche wenig befriedigende Ergebnisse erzielt. Die Brombestimmung, die Gensler5) benutzte, glaubte ich nicht prüfen za mïssen, da die neue Methode von $W$ ü $n s c h \theta$ aus demselben Züricher Institut kommt, aus dem Genslers Mitteilungen stammen. Die Angaben von Pilla $t^{6}$ ) erschienen erst, als meine Versuche weit vorgeschritten waren.

3) Guareschi, Neue, sehr scharfe, auch in Gegenwart anderer Halogene brauchbare Reaktion des Broms. Zeitschr. f. analyt. Chem. 1913, Bd. 52, S. 451.

4) A athenrieth, Die Bestimmung und Verteilung des Brom in Organen und im Blute nach Einnahme von Bromnatrium. Münch. med. Wochenschr. 1918, Bd. 65, S. 33.

5) Gensler, Über die Wirkung der Hypnotika (Neuronal) bei normalen und bei psychisch erregten Zuständen. Arch. f. exp. Path. u. Pharm. 1914, Bd. 77, S. 164.

6) Pilla t, Über einige Versuche, Brom in normalen menschlichen Organen nachzuweisen. Zeitschr. f. physiol. Chem. 1919, Bd. 108, S. 158. 
Entwicklungsgefäß aufgehängten, mit $\mathrm{SO}_{2}$-Fuchsin getränkten, stärkefreien Filtrierpapierstreifen einwirken ließ. Wunsche glaubte, um die änßerst empfindliche Reaktion zur Beantwortung quantitativer Fragen heranziehen zu können, den Vorgang der Br-Entwicklung and die Reaktion des freien $\mathrm{Br}$ mit dem $\mathrm{SO}_{2}$-Fuchsin trennen und deshalb in zwei verschiedene Gefäße verlegen zu müssen. Um das $\mathrm{Br}$ von dem Entwicklungsgefä $\beta$ in das eigentliche Reaktionsgefä $B$ überzuleiten, benutzte er einen Luftstrom von bestimmter Stärke und Daner. Die Einzelheiten des Verfahrens hier anzugeben, würde zu weit fuhren, es sei auf das Original selbst verwiesen.

Diese Methode hat in Wünsches eigner Hand und bei Alday ${ }^{1}$ ) ausgezeichnete Resultate ergeben. Aber schon Alday, der die Methode unmittelbar von Wünsche tủbernahm, betont die Schwierigkeiten, mit denen die Ausführung des Verfahrens verbunden ist. Ich habe diese Schwierigkeiten: Herstellung gleichmäßiger, dauerhafter, genaner Vergleichslösungen, Verhinderung des Ausflockens des Reaktionsproduktes, Bestimmung der Beendigung der Reaktion - bloB um einige kurz zu erwähnen - trotz genauer Einhaltung der Vorschrift ${ }^{2}$ ) so groB empfunden, daB ich nach tiber mehrere Wochen sich erstreckenden Vorversuchen die Anwendung der Methode von. Wunsche aufgab. Über ihren analytischen Wert vermag ich daher kein Urteil abzugeben, doch scheint mir die Brauchbarkeit einer Methode im wesentlichen abhängig zu sein von der Leichtigkeit, mit der sie reproduziert werden kann.

Es bleibt $W \mathfrak{u}$ sches Verdienst, erkannt zu haben, daß die Empfindlichkeit und Feinheit der Guareschischen Reaktion, die auch mich bestimmten, die Reaktion als Ausgangspunkt einer neuen Bestimmungsart beizubehalten, zur quantitativen Ausnutzung hervorragend geeignet ist. Meine Erfahrungen mit der Luftdurchleitung führten mich zuerst daza, die Reaktion der Br-Entwicklung und Farbstoffbildung wieder in einem Gefäß sich abspielen zu lassen.

1) Alday, Beiträge zur Theorie der Narkose. Arch. f. exp. Path. u. Pharm. 1919, Bd. 84, S. 339.

2) Bei der Herstellung der Fuchsinvorlagen hat Einhalten der Wünscheschen Vorschriften übrigens wenig Zweck. Unter dem Namen Fuchsin geht im Handel so verschiedene Ware, daß man nie mit den gleichen Lösungen arbeiten wird, mit denen der Autor seine Reaktion angestellt hat. Prinzipiell ist das natirlich belanglos, da es sich im Grunde doch um eine vergleichende Methode handelt. Es sei nur deshalb daranf hingewiesen, weil durch den genannten Umstand sich vielleicht der Widersprach erklärt, der zwischen $W$ it n s che, der 6 Atome $\mathrm{Br}$ auf ein Molekül Fuchsin rechnet, und $\mathrm{G}$ ureschi, der eine Tetrabromverbindung annimmt, besteht. 
Dazu mußte auf ein Oxydationsmittel zurtickgegriffen werden, das durch relative Farblosigkeit die Erkennung der violetten Br-Fuchsinverbindung nicht störte. Ein solehes bot sich im Chlorwasser dar, bei dessen Verwendung folgendes festgestellt wurde. Gibt man zu einer durch $\mathrm{H}_{2} \mathrm{SO}_{4}$ angesäuerten $\mathrm{NaBr}$-Lösung $1 \mathrm{ccm}$ einer $0,1 \%$ igen $\mathrm{SO}_{2}$-Fuchsinlösung, dann tropfenweise Chlorwasser, so wird die Flussigkeit intensiv violett, bei weiterem Cl-Zusatz grauviolett und schlägt bei einem gewissen Überschuß von $\mathrm{Cl}_{2}$ deutlich in goldgelb $\mathrm{um}$.

Men

\section{chemischen Vorgang}

bei der Reaktion haben wir uns dabei so zu denken: $\mathrm{Cl}_{2}$ setzt aus $\mathrm{HBr}$ molekulares $\mathrm{Br}$ in Freiheit, das mit dem Fuchsin sofort $\mathrm{Br}$-Fuchsin bildet. Bei Anwesenheit von weiterem $\mathrm{Cl}_{2}$ wird, wahrscheinlich auf Grund von Massenwirkungen, das $\mathrm{Br}$ auch aus seiner Verbindung mit dem Fuchsin vertrieben und gleichzeitig Chlorfuchsin gebildet. $\mathrm{Da}$ uber die Art der Verbindung zwischen $\mathrm{Br}$ und Fuchsin chemisch noch nichts ausgesagt werden kann, ist es schwierig, bei dem beschriebenen Vorgang zu zahlenmäßigen, exakten Vorstellungen zu gelangen. Ich möchte annehmen, daß sich in jedem gegebenen Fall zwischen violettem Brom-Fachsin und goldgelbem Chlor-Fachsin ein Gleichgewichtszustand ausbildet, der von dem Mengenverhältnis vorhandenen Chlors und Broms abhängig ist und im Moment des Utberwiegens von $\mathrm{Cl}_{2}$ ganz auf seiten des Chlorfachsins ruht. Die Erfahrung lebrt jedenfalls, daß, je mehr $\mathrm{Br}$ in der Lossung, desto mehr $\mathrm{Cl}$ bis zum Erscheinen des reingelben, violettfreien Farbentons erforderlich ist. Es lag nahe, diese Beobachtung einer >Titration * des Broms durch $\mathrm{Cl}_{2}$ mit dem Fuchsin als Indikator zugrunde zu legen. Jedoch ist die Reaktionsgeschwindigkeit besagten Vorgangs außerordentlich langsam. Beim Versuch, die Reaktion in der Wärme (Wasserbad $40-60^{\circ}$ ) vorzunehmen, erhält man durch die erhöhte Dampfspannung des $\mathrm{Br}$ ganz inkonstante Werte für den $\mathrm{Cl}_{2}$-Verbrauch. Eine Titration ließ sich also nicht durchfthren, doch war daran zu denken, die einzelnen Momente des zeitlichen Ablaufs einer Titration von dem Moment der BrFuchsinbildung bis zu dem Augenblick, wo nar noch Chlor-Fuchsin vorhanden ist, in mehreren einzelnen Reagensgläsern festzuhalten, indem man za gleichen Br-Mengen in jedes Glas verschiedene, und zwar steigende Mengen $\mathrm{Cl}_{2}$ setzte. Aus der. Chlormenge, die für das erste gerade ausgesprochen gelb erscheinende Glas verbraucht worden war, hătte man die gesuchte Brommenge tabellarisch oder rechnerisch bestimmen können. Leider bin ich aber weder rein empirisch, noch auf Grund theoretischer Überlegungen zur Aufstellung in diesem Sinne verwendbarer Gesetzmäßigkeiten gekommen. Die chemischen Vorgänge sind hier zu mannigfaltig, um sie differenzieren zu können. Der Einfluß von Nebenreaktionen, die beispielsweise durch die Gegenwart von $\mathrm{SO}_{2}$, durch das Entstehen von Chlorbrom bedingt sind, ist vorerst nicht za ubersehen.

Da mir vor allem daran gelegen war, auf möglichst kurzem Wege zu einer für biologische Fragen brauchbaren Bestimmung 
Eine neue Methode zur Bestimmung des Broms in kleinsten Mengen.

kleiner Mengen $\mathrm{Br}$ zu gelangen, habe ich die Versuche in dieser Richtung vorläufig aufgegeben und das

\section{Prinzip der Methode}

in einen Vergleich zwischen dem Verhalten der za prufenden und einer bekannten Bromlösung verlegt. Erhielt ich mit einem bestimmten Volumen einer unbekannten Bromidlösung bei Zusatz von wechselnden, steigenden $\mathrm{Mengen} \mathrm{Cl}_{2}$ eine Farbenreihe von violett bis gelb, so mußte notwendigerweise der Br-Gehalt mit dem einer bekannten Bromlösung thbereinstimmen, die unter gleichen Versuchsbedingungen mit den gleichen $\mathrm{Cl}_{2}$-Beträgen dieselbe Farbenreihe ergab. Die Herstellung einer Farbenreihe, bei geeigneten Mengenverhältnissen $\mathrm{Cl}_{2}$ und Bromid, ist nicht schwer. Mit 3-4 Reagensgläsern mit gleichem Volumen einer $\mathrm{NaBr}$-Lösung gewinnt man, bei richtiger Wahl, immer durch Zusatz gleichmäßig steigender Mengen des Oxydationsmittels eine Skala, die von Violett über die Übergangsfarben za gelb führt. Die richtige Wahl kann man bequem treffen, wenn man zu Beginn der Analyse uber den Chlorgehalt des Oxydans einigermaßen orientiert ist, wozu eine zahlenmäßige, etwa jodometrische Feststellung nicht einmal nötig ist. Auf welch einfache Weise diese Vorpriffung zweckmäßig vorzunehmen ist, wird bei der Ausfuhrung beschrieben.

Die Hauptschwierigkeiten, um einen einwandfreien Vergleich vornehmen zu können, lagen vielmehr in dem Umstand, daB die zu prufende Lösung eine Anzahl anderer Salze enthalten konnte, daß sie also nicht unter denselben Versuchsbedingungen stand, wie die Vergleichslösung. Doch ließ sich feststellen, daß die meisten Salze (der Ausnahme der Nitrite wird an anderer Stelle gedacht) fur den Reaktionsausfall belanglos sind. Nur die Chloride sind wieder wie bei den meisten Bromanalysen - die Ursache von Fehlern. Freilich äußert sich dieser Fehlerfaktor hier in umgekehrtem Sinn. Während wir bei anderen Methoden, wo wir $\mathrm{Cl}$ als $\mathrm{Br}$ mit analysieren, zu hohe Br-Werte erhalten, bedingt im vorliegenden Verfahren die Anwesenheit von $\mathrm{Cl}$ zu kleine Resultate. Zwei Ursachen durften daftir verantwortlich gemacht werden. Die Chloride werden durch $\mathrm{H}_{2} \mathrm{SO}_{4}$ ebenso wie $\mathrm{NaBr}$ in seine Halogenwasserstoffprodukte (HCl) verwandelt und dieses $\mathrm{muß}$ die Dissoziation des $\mathrm{HBr}$ zurïckdrängen und zweitens wird durch die Einwirkung von unterchloriger Säure, die sich sowohl im ursprtinglich verwandten Chlorwasser, wie in dem später benutzten $\mathrm{Ca}\left(\mathrm{OCl}_{2}\right.$ findet, aus $\mathrm{NaCl}: \mathrm{Cl}_{2}$ entstehen. Es ist klar, daß dadurch im Sinne des oben besprochenen Vorgangs - weil eben mehr $\mathrm{Cl}_{2}$ anwesend ist - die Bildung des Chlorfuchsins bei 
einem geringeren Oxydanszusatz eintritt, als bei einer gleichstarken, aber NaCl-freien Bromvergleichslösung. Es ist mir nicht gelungen, den Fehler so zahlenmäßig zu erfassen, um eine entsprechende Korrektur am Resultat anbringen zu können. Der Fehler steht weder mit dem absoluten Chlorgehalt, noch mit dem Verhältnis $\mathrm{Cl} / \mathrm{Br}$ in Proportion. Innerhalb gewisser Grenzen ist aber erfahrungsgemäß der Fehler klein genug, um vernachlässigt werden zu können. Nach meinen Erfahrungen wird chloridfreies Material zweifellos am besten mit reiner $\mathrm{NaBr}$-Lösung verglichen. Für Bestimmungen in kochsalzhaltigem Analysenmaterial mit NaCl-Mengen, wie sie die physiologischen Ausgangsstoffe mit sich bringen, hat sich mir eine Vergleichslösung am besten bewährt, die die 10 fach äquimolore Menge des Bromids an Chloriden enthält.

An

werden somit gebraucht:

\section{Reagentien}

1. Vergleichslösung $\mathrm{I}$ : enthält in $1 \mathrm{ccm} 1 \mathrm{mg} \mathrm{Br}$, in $1 \mathrm{H}_{2} \mathrm{O}$ $12,9 \mathrm{~g}$ chemisch reines $\mathrm{NaBr}$. Vergleichslösung II: ebenfalls in $1000 \mathrm{ccm} \mathrm{Aq}$. dest., $0: 12,9 \mathrm{~g} \mathrm{NaBr}$ und $7,3 \mathrm{~g} \mathrm{NaCl}$.

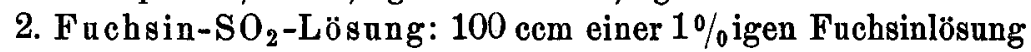
werden $80-90 \mathrm{mg}$ frisch bereitetes, in Wasser aufgenommenes $\mathrm{SO}_{2}$ zugesetzt; das ganze auf $1000,0 \mathrm{ccm}$ anfgefullt. Die Flitssigkeit wird nach 24-36 Stunden farblos 1 ) oder blaß gelblich und bält sich, kuhl und dunkel aufbewahrt, wochenlang.

3. Oxydationsmittel. Statt des Chlorwassers, dessen Unbeständigkeit störend wirkt, benutze ich jetzt das Kalksalz der unterchlorigen Säure, das die chemische Fabrik »Griesheim Elektron * unter dem Namen „Caporit* in Handel bringt und von dem sie mir Proben in dankenswerter Weise überlassen hat. $1,0 \mathrm{~g}$ des leichtlöslichen, etwas tuber $40 \% \mathrm{Cl}$ enthaltenden Pulvers, wird in 500,0 ccm Aq. dest. gelöst und filtriert.

4. $25 \% \mathrm{H}_{2} \mathrm{SO}_{4}$. ( $\mathrm{HCl}$ kann aus den bei Besprechung des Chlorfehlers hervorgehenden Grunden nicht benutzt werden. Außerdem enthält die Salzsäure des Handels - für den käuflichen Chlorkalk. gilt dasselbe - stets etwas $\mathrm{Br}$.)

5. Analysenmaterial. Die zu untersuchende Lösung enthalte das Brom als Bromalkali. Organische Stoffe werden deshalb alkalisch

1) Das Fuchsin skleine Krystalle erhielt ich durch Herrn Kertess von der Firma Casella \& Co., Mainkur, wofür ihm auch hier bestens gedankt sei. Verwendet man unreines Fuchsin, läuft man in Gefahr, daß sich die Lösung nur mangelhaft oder noch langsamer entfärbt. 
ohne Zusatz von $\mathrm{Na}_{2} \mathrm{O}_{2}$ und Salpeter verascht. Die Anwesenheit auch nur der geringsten Spuren Peroxyd oder Nitrit verhindert das Zustandekommen der Bromfuchsinverbindung. Alkoholische Lösungen sind unbrauchbar, da Aldehyde - wie Guareschi schon angibt eine Br-Reaktion vortäuschen. Die Brom-Konzentration der zu untersuchenden Lösung (weiterhin X genannt) liegt zwischen 0,01 und $0,1 \%$.

Der Übersicht halber teile ich die

\section{Ausführung der Bestimmung}

in vier Teile ein. Der erste Teil dient der bereits erwähnten, empirischen Feststellung der Stärke von $\mathrm{C}$ (Caporitlösung), bezüglich seiner Fähigkeit $\mathrm{Br}$ frei zu machen und aus dem Bromfuchsin Chlorfuchsin entstehen zu lassen. Die angegebene C-Lösung ist so beschaffen, daß mit $0,1 \mathrm{mg}$ Br bei einem* Zusatz von 0,2 oder 0,25 oder $0,3 \mathrm{ccm} \mathrm{C}$ einmal ein deutlich grauvioletter Farbenton entsteht. Es wird zu 3 Reagensgläsern mit je $0,1 \mathrm{ccm}$ Vergleichslösung je $1 \mathrm{ccm}$ Säure, dann zu den einzelnen Gläsern die genannten Volumina C, die man aus einer Mikrobürette mit $0,05 \mathrm{ccm}$ Skalenteilung zufließen läßt, zugesetzt. SchlieBlich kommt zu jedem Glas $1 \mathrm{ccm}$ Fuchsin- $\mathrm{SO}_{2}$. Eine der Proben wird grauviolett.

Mit dem Volumen von C, das den Übergangston ergeben hat, wird im $\mathrm{zw}$ eiten Teil die approximative Bestimmung des $\mathrm{Br}$ in $\mathrm{X}$ vorgenommen, indem wir diese Menge $C$ nacheinander mit 0,1, 0,2, $0,5,0,8$ und wenn notwendig $1,0 \mathrm{ccm}$ von $X$ zusammenbringen, bis ein grauvioletter Ton auftritt oder eine Probe violett wird. (Die Reihenfolge der übrigen Zusätze ist ein für allemal dieselbe.) Haben wir so einen grauvioletten Reaktionsausfall erhalten, so wissen wir, $\mathrm{daB}$ in dem betreffenden Volumen ungefähr $0,1 \mathrm{mg} \mathrm{Br}$ sein wird. Fehlt der Übergangston und ist ein Inhalt gelb geworden, die nächste Probe violett, so ist klar, daß der Übergangston zwischen den zwei angewandten Volumina liegen muß. Ist $0,1 \mathrm{ccm}$ schon violett, ist $\mathrm{zu}$ viel $\mathrm{Br}$ in $\mathrm{X}$; die Lösung ist zu verdinnen. Ist 1,0 noch ausgesprochen gelb, so ist weniger als $0,1 \mathrm{mg}$ Br vorhanden; ein Fall, der gesondert besprochen werden muß.

So kennen wir annähernd die Beziehungen von $\mathrm{X}$ und $\mathrm{Cl}_{2}$. Im dritten Teil wird zar Anstellung des Vergleichs mit dem aus Teil 2 sich ergebenden Volumen eine Farbenreihe - violett, grauviolett, gelb - hergestellt, die durch Zusatz wechselnder Mengen $\mathrm{C}$ entsteht. Beispielsweise: $0,25 \mathrm{ccm} \mathrm{C}$ hat mit $0,1 \mathrm{mg} \mathrm{Br}$ grauviolett gegeben, $0,3 \mathrm{ccm} X$ mit $0,25 \mathrm{ccm} C$ desgleichen, dann muß $0,3 \mathrm{ccm} X$ mit $0,2 \mathrm{ccm} C$ violetten und mit $0,3 \mathrm{ccm} C$ gelben Reaktionsansfall ergeben. 
Im vierten Teil werden solche Farbenreihen - ohne das Analysenmaterial nochmals in Anspruch zu nehmen - mit steigenden Mengen der Vergleichslösung - 0,1, 0,125, 0,15, 0,175, 0,2 usw. mg Br - die man mit einer in $1 / 100 \mathrm{ccm}$ geteilten Pipette der Vorratsflasche entnimmt, hergestellt. Durch Nebeneinanderhalten der Farbenreihen von $X$ ans Teil 3 and den entsprechenden ans Teil 4 kann dann abgelesen werden, daB soundsoviel ccm $X$ mehr oder weniger $\mathbf{m g ~} \mathbf{B r}$ enthalten als 0,1 bzw. $0,125 \mathrm{mg} \mathrm{Br}$ usw.

Ein

\section{Beispiel}

mag den Hergang deutlicher illustrieren.

Kum Zwocke der leichteren Verständigung und Vereinfachnng des Beispiels - aber nur zu diesem Zwecke - führe ich hier eine Zeichensprache ein. Es soll heißen: $+=$ violett, $-=$ gelb, $\pm=$ grauviolett. Mit Einklammerungen und Fragezeichen werden noch feinere Schattierungen ausgedrtickt. Es ist aber festzuhalten, daß stets die Gläser und Farben selbst verglichen werden müssen, nur so werden kleine Nuanceunterschiede erkannt. Die Farbenreihen mit gleichen Zeichen haben keineswegs den gleichen Bromgehalt, sondern können geringe Mengen mehr oder weniger enthalten, was bei direktem Vergleich nicht entgeht.)

\section{Filtrat $14,5 \mathrm{ccm}$.}

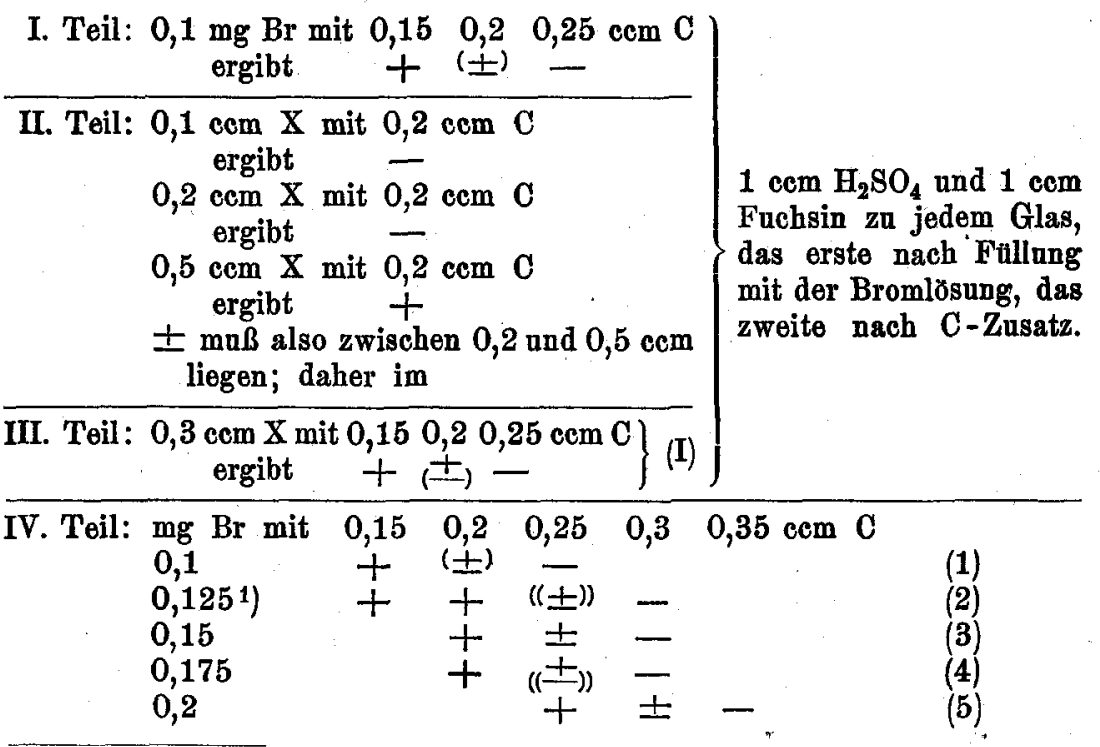

1) Die Empfindlichkeit der Reaktion geht schon aus den Farbenunterschieden hervor, die Differenzen von $0,025 \mathrm{mg}$ hinterlassen. 
Vergleichen wir jetzt Reihe (I) mit (1) und (2), so erhellt aus den Gläsern mit $0,2 \mathrm{cem} \mathrm{C}-\mathrm{Zusatz}$, daß (I) mehr $\mathrm{Br}$ enthält als (1) oder in $0,3 \mathrm{ccm} \mathrm{X}$ mehr als $0,1 \mathrm{mg} \mathrm{Br}$ sind; ferner, daß (I) weniger als (2) $\mathrm{Br}$ besitzt, mithin in $0,3 \mathrm{ccm} \mathrm{X}$ weniger als $0,125 \mathrm{mg} \mathrm{Br}$ sind.

Ergebnis: $0,3 \mathrm{ccm} X=0,1-0,125 \mathrm{mg} \mathrm{Br}$.

Im Mittel, wie wir mit hinreichender Genauigkeit für physiologische Zwecke meist rechnen dürfen:

$0,3 \mathrm{ccm}=0,112 \mathrm{mg} \mathrm{Br} ; 1 \mathrm{ccm}=0,37^{3} \mathrm{mg} ; 14,5 \mathrm{ccm}=5,4^{1} \mathrm{mg} \mathrm{Br}$. Gegeben waren: $5,5^{9} \mathrm{mg} \mathrm{Br}(7,2 \mathrm{mg} \mathrm{NaBr})$.

In dem hier durchgeführten Beispiel liegen die Verhältnisse insofern glinstig, als die Interpolation zwischen 0,1 and $0,125 \mathrm{mg} \mathrm{zu}-$ fällig in die Nähe des wirklichen Wertes $0,3 \mathrm{ccm}=0,11^{5} \mathrm{mg} \mathrm{Br}$ führt. Der wahre Br-Wert könnte, ohne daß es in unserem Ergebnis zum Ausdruck kommt, näher an 0,1 oder näher an $0,125 \mathrm{mg}$ statt fast in der Mitte liegen. Das Gesamtresultat würde dann, da die Einzelbeobachtang in unserem Fall mit $\frac{14,5}{0,3}=48,3$ multipliziert werden muß, unter anderen Bedingungen wesentlich schlechter ausfallen. Je kleiner die Gesamtbrommenge, desto kleiner natürlich der Fehler für das Endergebnis. Wenn es bei größeren Mengen aber auf größere Genauigkeit ankommt, könnte man den Fehler dadurch verkleinern, $\mathrm{daß}$ man, statt mit $0,1-0,2 \mathrm{mg} \mathrm{Br}$ zu arbeiten, größere Mengen nimmt. Aber das hat seine Grenze, weil, je höher man taber $0,2 \mathrm{mg}$ Br hinansgeht, der Vergleich dadurch ungemein erschwert wird, daß unter Beibehaltung der $\mathrm{Cl}_{2}$-Konzentration und der Abstände von $0,05 \mathrm{ccm}$ sich die Zahl der Gläser vermehrt, die Übergangstöne enthalten. Konzentriert man die Chlorlösung oder steigt man beim $\mathrm{Ca}(\mathrm{OCl})_{2}-\mathrm{Zusatz}$ in größeren Raumteilabständen, so leidet wiederum die Empfindlichkeit der Bestimmung and nichts wäre damit gewonnen.

Man kann jedoch den erwähnten Fehler sehr gut herabsetzen and die Annäherung an den wahren Wert erhöhen, wenn man bei ausreichendem Analysenmaterial noch weitere Farbenreihen mit jeweils um $1 / 10$ cem größerem Volumen von $X$ darstellt und die vorhin rechnerisch ausgefuhrte Interpolation auf diese Weise sich experimentell selber regeln läßt.

$\mathrm{Zu}$ diesem $\mathrm{Z}_{\text {weck }}$ werden im III. Teil noch Farbenreihen mit 0,4 und $0,5 \mathrm{ccm}$ ron $X$ angefertigt.

$$
\left.\begin{array}{cccccc}
0,4 \mathrm{ccm} X \text { mit } & 0,15 & 0,2 & 0,25 & 0,3 \mathrm{~cm} \mathrm{C} \\
+ & + & \pm & - & \\
0,5 \mathrm{~cm} X \text { mit } & 0,15 & 0,2 & 0,25 & 0,3 & 0,35 \mathrm{~cm} \mathrm{C} \\
+ & + & \pm & -
\end{array}\right\}
$$


Vergleicht man (II) mit (3) und (4), so ergibt sich - was nicht aus den Zeichen (vgl. den Kleindruck zu Beginn des Beispiels) aber aus dem unmittelbaren Vergleich hervorgeht - daß 0,4 ccm X etwas mehr als 0,15 und weniger als $0,175 \mathrm{mg} \mathrm{Br}$, daß ferner aus Vergleich von (III) mit (4) und (5) $0,5 \mathrm{ccm} X$ mehr als 0,175 und weniger als $0,2 \mathrm{mg} \mathrm{Br}$ enthalten. Damit sind uns 3 Ungleichungen gegeben:

$$
\begin{aligned}
& 0,1 \mathrm{mg} \text { Br } \leq 0,3 \mathrm{ccm} \mathrm{X}<0,125 \mathrm{mg} \mathrm{Br}
\end{aligned}
$$

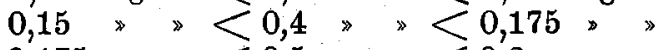

$$
\begin{aligned}
& 0,175 \text { " " }<0,5 \text { * " }<0,2 \text {, " }
\end{aligned}
$$

die wir auf $0,1 \mathrm{ccm}$ umrechnen

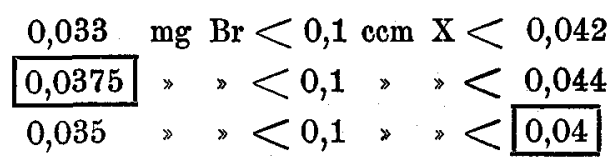

Die sich am nächsten liegenden Werte der linken und rechten Seiten milssen dem wahren Wert sich am besten nähern.

$$
\begin{aligned}
& 0,1 \mathrm{~cm}=0,0375-0,40 \mathrm{mg} \mathrm{Br} ; \text { im Mittel für } 1,0 \mathrm{ccm} \\
& 1,0 *=0,38^{7} \mathrm{mg} \mathrm{Br}
\end{aligned}
$$

für 14,5 » $=5,61$ »

Der wahre Wert unseres Beispiels ist $0,38^{5}$ bzw. $5,5^{9} \mathrm{mg}$, dem wir uns auf diese Weise erheblich mehr genähert haben, als mit der einmaligen Beobachtung mit dem Ergebnis 0,373 bzw. 5,41 $\mathrm{mg} \mathrm{Br}$. (Statt des Mittelwerts ziehe ich vor, die beiden Grenzwerte anzugeben.)

Einer besonderen Erwähnung bedarf noch der Zeitpunkt, in dem die Farbenreihen verglichen werden sollen. Angesichts der bereits erwähnten geringen Reaktionsgeschwindigkeit, darf nicht umgehend nach dem Fuchsinzusatz der Vergleich vorgenommen werden. Der Endzustand des chemischen Vorgangs ist immer abzuwarten. Andererseits dürfen ältere Farbenreihen nicht mit frischen verglichen werden. Die Farblösung hält sich nämlich nicht, sie flockt aus, so daß man nach Stunden zu Boden sitzende, flockige Farbstoffteilchen und eine überstehende, farblose, klare Flüssigkeit vor sich hat. Es geht natürlich nicht an, zwischen eben hergestellten und ausflockenden oder ausgeflockten Farbenreihen Vergleiche zu ziehen. Man vergleiche also Farbenskalen innerhalb 20 Minuten nach ihrer Herstellung. Da jedoch die Farbflocken ganz ansgezeichnet die einzelnen Farbqualitäten wiedergeben, ist es auch möglich, den Vergleich nach 12 bis 24 Stunden anzustellen. Man verfährt dabei genau so wie bei den Lösungen, nur ist Vorsorge zu treffen, daß man beim Ansetzen des 
Versuchs alle etwa in Betracht kommenden Reihen von 0,1 bis 0,2 oder $0,3 \mathrm{Br}$ fehlerlos anfertigt. Eine Nachlieferung eines oder mehrerer Gläser zur Vervollständigung ist nicht statthaft, da in dem inzwischen verstrichenen Zeitraum die Caporitlösung sich mit Sicherheit etwas verändert hat und damit die Versuchsbedingungen geändert sind. In solchen Fällen wäre die ganze Analyse za wiederholen.

Bei einer Konzentration von $0,1 \mathrm{mg}$ in $1 \mathrm{ccm}$ braucht man $\mathrm{zur}$ Bestimmung wenigstens $6-7 \mathrm{ccm}$, was dann einem Gesamtgehalt von $0,6-0,7 \mathrm{mg} \mathrm{Br}$ entspricht. Da man aber, wenn statt $1 \mathrm{ccm}$ Fuchsin nur 0,5 ccm zugesetzt werden und auch die Menge des Oxydans auf 0,05 und $0,1 \mathrm{ccm}$ hernntergeht, noch im Notfall Farbenreihen mit $0,04,0,05$ und $0,075 \mathrm{~g} \mathrm{Br}$ erhält, kann man gelegentlich auch noch eine Gesamtmenge von $0,4 \mathrm{mg} \mathrm{Br}$ als unterste Grenze quantitativ erfassen.

Der Umstand, daß bei meiner Methode verschiedene Farbdichten verglichen werden, da ja Vergleichslösung und zu prüfende Lösung in verschiedenen Volumina verwandt werden, ist nach meiner Erfahrung belanglos. $\mathrm{Zu}$ beiden Vergleichsobjekten kommt soviel Flüssigkeit (Säure, Fuchsin und Caporit), daß der Unterschied in der Farbdichte praktisch ausgeglichen wird.

Schließlich sei noch hervorgehoben, daß der quantitative Nachweis ${ }^{1)}$ von $\mathrm{Br}$ bei einer Verdünnung von 2:100000 in $1 \mathrm{ccm}$ gut gelingt. (Walter ${ }^{2}$ ) gibt mit der Guareschischen ${ }^{3}$ ) Originalmethode $1: 10000$ an.) Man ändert für diesen Fall die Reihenfolge der Zusätze, indem man der angesäuerten Br-Lösung erst 0,4 ccm Fachisin$\mathrm{SO}_{2}$, dann tropfenweise Caporit zusetzt. Bei $0,02 \mathrm{mg} \mathrm{Br}$ in $1 \mathrm{ccm}$ entsteht ein deutlicher violetter Ring.

Zum Schluß folgen einige Analysenresultate. Auf Wiedergabe weiterer, ausfuhrlicher Beleganalysen muß ich, da die Methode kurze, eindeutige Protokolle nicht gestattet, angesichts der derzeitigen Raumnot verzichten.

Die Anwendung der Methode wird vor allem fur kleine $\mathrm{Br}-$ Mengen angezeigt sein, denn erstens versagen für diese die übrigen Bestimmungen und zweitens liefert sie, wie aus der Tabelle ersichtlich, gerade bei Mengen von 2-12 mg, die glinstigsten Resultate.

1) Daß Walter ${ }^{2}$ ) jüngst auch im Goldchlorid ein empfindliches Reagens auf $\mathrm{Br}$ gefunden hat, sei erwähnt.

2) Walter, Untersuchungen über die Permeabilität der Meningen. Zeitschr. f. d. ges. Neurol. 1919, Bd. 47, S. 380.

3) Guareschi, s. S. 18 . 


\begin{tabular}{|c|c|c|c|c|c|}
\hline $\begin{array}{l}\text { Ver- } \\
\text { such } \\
\text { Nr. }\end{array}$ & Material & $\begin{array}{l}\text { Filtrat } \\
\text { bzw. } \\
\text { Lösung } \\
\text { in ccm }\end{array}$ & $\begin{array}{l}\text { Gefunc } \\
\text { Grenzwerte } \\
\text { in } \mathrm{mg}\end{array}$ & $\begin{array}{l}\text { den Br } \\
\begin{array}{c}\text { Mittelwerte } \\
\text { in } \mathbf{~ m g}\end{array}\end{array}$ & $\begin{array}{c}\text { Gegeben } \\
\mathrm{Br} \\
\text { in } \mathrm{mg}\end{array}$ \\
\hline 5 & $15 \mathrm{mg} \mathrm{N} \mathrm{N}_{4} \mathrm{Br}$ in $10 \mathrm{ccm}$ Serum & 9,5 & - & $\mathbf{1 1 , 5}$ & 12,2 \\
\hline 6 & $\begin{array}{l}4,4 \mathrm{mg} \text { Bromhexahydrobenzol- } \\
\text { süureamid }\end{array}$ & 4,2 & 一 & 1,68 & 1,71 \\
\hline 7 & $\begin{array}{l}\text { 6,5 mg Bromhexahydrobenzol- } \\
\text { säureamid }\end{array}$ & 6,5 & $一$ & 2,1 & 2,52 \\
\hline$W_{0}$ & $\begin{array}{l}71,0 \mathrm{mg} \mathrm{NaBr} \text { in physiologi- } \\
\text { scher NaCl-Lösung }\end{array}$ & 26,8 & 一 & 56,9 & 55,2 \\
\hline $\mathrm{W}_{1}$ & $\begin{array}{l}8,8 \mathrm{mg} \mathrm{NaBr} \text { in physiologischer } \\
\mathrm{NaCl}-\mathrm{Lösung}\end{array}$ & 17,4 & - & 6,2 & 6,9 \\
\hline$W_{2}$ & $\begin{array}{l}7,43 \mathrm{mg} \mathrm{NaBr} \text { in physiologi- } \\
\text { scher } \mathrm{NaCl}-\mathrm{Lösung}\end{array}$ & 16,3 & $5,73-6,56$ & 6,14 & 5,77 \\
\hline $\mathbf{W}_{3}$ & $\begin{array}{l}4,0 \mathrm{mg} \mathrm{NaBr} \text { in } \mathrm{NaCl}-\mathrm{Lösung} \\
\text { unbekannter Konzentration }\end{array}$ & 24,5 & $3,06-3,43$ & 3,24 & 3,1 \\
\hline $\mathrm{W}_{4}$ & $\begin{array}{c}13,0 \mathrm{mg} \mathrm{NaBr} \text { in } \mathrm{NaCl}-\mathrm{Lösung} \\
\text { unbekannter Konzentration }\end{array}$ & 20,4 & $10,1-10,5$ & 10,3 & 10,0 \\
\hline$W_{5}$ & $\begin{array}{c}15,0 \mathrm{mg} \mathrm{NaBr} \text { in } \mathrm{NaCl}-\mathrm{Lösung} \\
\text { unbekannter Konzentration }\end{array}$ & 30,5 & $11,4-12,2$ & 11,8 & 11,7 \\
\hline $\mathbf{P}_{1}$ & $44,0 \mathrm{mg} \mathrm{NaBr}$ in Aq. dest. & 50,0 & $28,7-35,1$ & 31,9 & 34,2 \\
\hline $\mathrm{W}_{6}$ & $\begin{array}{l}9,66 \mathrm{mg} \mathrm{NaBr} \text { in etwa } 15 \% \\
\mathrm{NaCl} \text {-Lösung }\end{array}$ & 15,0 & - & 6,6 & 7,5 \\
\hline $0_{1}$ & $\begin{array}{l}20,4 \mathrm{mg} \mathrm{Br} \text { als } \mathrm{NaBr} \text { aus einer } \\
\mathrm{NaCl} \text {-freien Lösung }\end{array}$ & 39,5 & - & 19,2 & 20,4 \\
\hline $0_{2}$ & $\begin{array}{l}22,0 \mathrm{mg} \mathrm{Br} \text { als } \mathrm{NaBr} \text { aus einer } \\
\mathrm{NaCl} \text {-haltigen Lösung }\end{array}$ & 41,0 & $20,0-23,2$ & 21,75 & 22,0 \\
\hline $\mathrm{W}_{7}$ & $\begin{array}{l}16,9 \mathrm{mg} \mathrm{NaBr} \text { in } 0,73 \% \mathrm{NaCl}- \\
\text { Lösung }\end{array}$ & 14,8 & $7,45-8,19$ & 7,82 & 8,5 \\
\hline $\mathrm{W}_{8}$ & $16,9 \mathrm{mg} \mathrm{NaBr}$ in $\mathrm{Aq}$. dest. & 24,4 & $8,5-9,4$ & 8,9 & 8,5 \\
\hline$W_{9}$ & $3,3 \mathrm{mg} \mathrm{NaBr}$ in Frosch-Ringer & 8,0 & - & 2,58 & 2,5 \\
\hline$W_{10}$ & $9,9 \mathrm{mg} \mathrm{NaBr}$ in $\mathrm{Aq}$. dest. & 16,4 & $8,0-8,25$ & 8,1 & 7,7 \\
\hline
\end{tabular}

Anmerkung bei der Korrektur. Auf die ktirzlich erschienene Arbeit von G. Hartwich, »Eine neue Methode quantitativer Brombestimmung \&1) stieß ich erst währepd der Drucklegung meiner Arbeit. Die Hartwichsche Methode, die zum Teil in den Grundlagen mit der beschriebenen Methodik ubereinstimmt, scheint sehr gute Resultate zu ergeben. $\mathrm{Ob}$ sie sich auch für kleinste $\mathrm{Br}-\mathrm{Mengen}$ eignet, müßte noch geprift werden.

1) Bioch. Ztschr. 1920, Bd. 107, S. 202. 